\title{
Erratum to: High field magnetic resonance imaging of rodents in cardiovascular research
}

\author{
Laetitia Vanhoutte ${ }^{1,2} \cdot$ Bernhard L. Gerber $^{3,4} \cdot$ Bernard Gallez $^{5} \cdot$ Chrystelle Po $^{6}$. \\ Julie Magat $^{7} \cdot$ Jean-Luc Balligand $^{2} \cdot$ Olivier Feron $^{2} \cdot$ Stéphane Moniotte $^{1}$
}

Published online: 4 August 2016

(c) Springer-Verlag Berlin Heidelberg 2016

\section{Erratum to: Basic Res Cardiol (2016) 111:46 \\ DOI 10.1007/s00395-016-0565-2}

Unfortunately, the first name and surname of an author were accidentally exchanged in the original article.

The correct list of authors reads as follows:

Laetitia Vanhoutte, Bernhard L. Gerber, Bernard Gallez, Chrystelle Po, Julie Magat, Jean-Luc Balligand, Olivier Feron, Stéphane Moniotte

The authors apologize for any inconveniences caused.

The online version of the original article can be found under doi:10.1007/s00395-016-0565-2.

Laetitia Vanhoutte

laetitia.vanhoutte@uclouvain.be

1 Department of Paediatric Cardiology, Cliniques universitaires Saint Luc, Université Catholique de Louvain (UCL), Brussels, Belgium

2 Pole of Pharmacology and Therapeutics (FATH), Institute of Experimental and Clinical Research (IREC), Université Catholique de Louvain (UCL), Brussels, Belgium

3 Division of Cardiology, Cliniques universitaires Saint Luc, Université Catholique de Louvain (UCL), Brussels, Belgium

4 Pole of Cardiovascular Research (CARD), Institute of Experimental and Clinical Research (IREC), Université Catholique de Louvain (UCL), Brussels, Belgium

5 Biomedical Magnetic Resonance Unit (REMA), Louvain Drug Research Institute (LDRI), Université Catholique de Louvain (UCL), Brussels, Belgium

6 CNRS, ICube, FMTS, Institut de Physique Biologique, Faculté de Médecine, Université de Strasbourg, Strasbourg, France

7 L'Institut de Rythmologie et de Modélisation Cardiaque (LIRYC), Inserm U1045, Bordeaux, France 